\title{
Cirurgias seguras: elaboração de um instrumento de enfermagem perioperatória*
}

\author{
Safe surgery: elaboration of an instrument for perioperative nursing care \\ Cirurgías seguras: elaboración de un instrumento de enfermería perioperatoria
}

Edna Lopes Monteiro ${ }^{1}$, Cleuciane Lima de $\mathrm{Melo}^{2}$, Thatiana Lameira Maciel Amaral ${ }^{3}$, Patricia Rezende do Prado ${ }^{4}$

\begin{abstract}
RESUMO: Objetivo: construir e validar o conteúdo de um instrumento para registro da assistência de Enfermagem Perioperatória para um Hospital de Clínicas, visando a atender as exigências da Organização Mundial da Saúde propostas no 2. ${ }^{\circ}$ Desafio Global e reafirmadas no Manual Cirurgias Seguras Salvam Vidas do Ministério da Saúde Brasileiro. Método: trata-se de um relato de experiência. A construção do instrumento fundamentou-se na Teoria das Necessidades Humanas Básicas e na revisão bibliográfica sobre o tema. Para a validação de conteúdo, foram realizadas aulas expositivas para treinamento da Equipe de Enfermagem. Resultados: o instrumento foi analisado como satisfatório pelas avaliadoras por ser de fácil interpretação e preenchimento. Conclusão: ressalta-se a importância da parceria entre a academia e o serviço de saúde. Sugere-se que o desenvolvimento de instrumentos com esta finalidade seja realizado em conjunto, no intuito de auxiliar os profissionais nos campos de estágio, envolver alunos estagiários e valorizar a assistência de Enfermagem.
\end{abstract}

PALAVRAS-CHAVE: Enfermagem perioperatória. Diagnósticos de enfermagem. Segurança.

ABSTRACT: Objective: The purpose of this study is to develop and validate an instrument of perioperative nursing for a university hospital aiming to meet the requirements proposed at the 2nd Global Challenge of the World Health Organization (WHO) and reiterated in the "Safe Surgeries Save Lives" Manual from the Brazilian Ministry of Health. Method: It is an experience report of descriptive approach. The construction of the instrument was based on the Basic Human Needs Theory and on literature review. Training lectures were conducted with the nursing staff to validate the instrument contents. Results: The instrument was assessed as satisfactory by evaluators because it can be easily filled in. Conclusion: We realized how important the partnership between the academy and the health service is. We suggest that more studies such as this one be undertaken in order to assist the field of training, engage trainee students, and enable the recovery of our profession.

KEYWORDS: Perioperative nursing. Nursing diagnosis. Safety.

RESUMEN: Objetivo: construir y validar el contenido de un instrumento para registro de la asistencia de enfermería perioperatoria en un Hospital de Clínicas, con el objetivo de atender las exigencias de la Organización Mundial de Salud, propuestas en el $2^{\circ}$ Desafío Global y reafirmadas en el Manual "Cirugías Seguras Salvan Vidas" del Ministerio de Salud. Método: se trata de un relato de experiencia. La construcción del instrumento se fundamentó en la teoría de las Necesidades Humanas Básicas y en la revisión bibliográfica sobre el tema. Para la validación del contenido se realizaron clases expositivas, con el fin de entrenar al equipo de enfermería. Resultados: el instrumento fue evaluado como satisfactorio al ser de fácil interpretación y cumplimentación. Conclusión: se resalta la importancia de que haya colaboración entre el mundo académico y el servicio de salud. Se sugiere que el desarrollo de dicho instrumento se realice en colaboración, a fin de auxiliar a los profesionales en los ámbitos de pasantía, involucrar a los alumnos en prácticas y valorar la asistencia de enfermería.

PALABRAS CLAVE: Enfermería perioperatoria. Diagnósticos de enfermería. Seguridad.

${ }^{1}$ Discente. Monitora da Disciplina de Enfermagem em Centro Cirúrgico. Curso de Bacharelado em Enfermagem. Universidade Federal do Acre (UFAC).

E-mail: ednalops@hotmail.com

${ }^{2}$ Discente. Bolsista PIBIC/CNPq. Curso de Bacharelado em Enfermagem. Universidade Federal do Acre (UFAC). E-mail: cleuci_limah@hotmail.com

${ }^{3}$ Enfermeira. Professora Assistente. Universidade Federal do Acre (UFAC). Mestre em Saúde Coletiva. Doutoranda. Escola Nacional de Saúde Pública (ENSP). Fundação Oswaldo Cruz (FIOCRUZ). E-mail: thatianalameira27@hotmail.com

${ }^{4}$ Enfermeira. Professora Assistente. Universidade Federal do Acre (UFAC). Bacharel em Enfermagem. Escola de Enfermagem de Ribeirão Preto (EERP). Universidade de São

Paulo (USP). Especialista em UTI e Auditoria em Serviços de Saúde. Mestre em Saúde Coletiva. E-mail: patyrezende@terra.com.br

Alameda Atenas, 67, Condomínio Florença, Edifício San Marino, apto. 93. CEP 69.915-422. Jardim Europa II. Rio Branco, AC, Brasil.

Telefone: (68) 9971-8637. E-mail: patyrezende@terra.com.br

*Pesquisa realizada no Hospital das Clínicas do Acre. 


\section{Introdução}

Segundo a Organização Mundial de Saúde (OMS), no ano de 2008, foram realizadas cerca de 234 milhões de intervenções cirúrgicas, com média de uma cirurgia a cada 25 pessoas. Destas, estima-se que ocorreram sete milhões de complicações e dois milhões de mortes, sendo que 50\% dessas mortes e complicações eram evitáveis, o que sugere que, mesmo com o intuito de salvar vidas, as falhas de segurança nos procedimentos cirúrgicos podem causar danos importantes ao paciente, como a invalidez ou a morte ${ }^{1,2}$.

Nos países industrializados, as complicações importantes são relatadas em 3 a 16\% dos procedimentos cirúrgicos, com frequência de incapacidade permanente ou morte entre 0,4 a $0,8 \%$. Já nos países em desenvolvimento, a taxa de mortalidade durante as cirurgias de grande porte varia entre 5 e $10 \%$, e a mortalidade originada unicamente pela anestesia geral é relatada em uma frequência de uma em 150 pessoas na África subsaariana. As infecções e outras morbidades pós-operatórias também são uma grave preocupação em todo o mundo ${ }^{1}$.

No ano de 2002, devido ao crescente número de cirurgias e suas complicações, a OMS realizou a $55^{\mathrm{a}}$ Assembleia da Saúde Mundial, na qual foram discutidos temas relacionados à saúde do paciente. Os debates culminaram com a criação da Aliança Mundial para a Segurança do Paciente em 2004, cujo objetivo era intervir em temas relacionados à segurança do paciente. Para tanto, esta aliança lançou dois desafios globais, sendo que o segundo desafio, lançado nos anos de 2007 e 2008, objetivou a prevenção de infecções de sítio cirúrgico, a anestesia segura, as equipes cirúrgicas seguras e os indicadores da assistência cirúrgica ${ }^{1,2}$.

Para atender o segundo Desafio Global da OMS, o Ministério da Saúde Brasileiro, em parceria com a Organização Pan-Americana da Saúde (OPAS/OMS), criou um manual voltado para a segurança do paciente cirúrgico intitulado Cirurgias Seguras Salvam Vidas, o qual elencou quatro desafios subjacentes a serem vencidos para melhorar a segurança cirúrgica: 1) o reconhecimento da cirurgia como uma preocupação significativa em saúde pública; 2) o acesso à assistência cirúrgica básica como uma preocupação em cenários de baixa renda; 3 ) a realização de práticas de segurança já existentes e, no entanto, não utilizadas de maneira confiável em nenhum país, e 4) a prevenção de complicações anestésicas ${ }^{1}$.

Com o intuito de auxiliar na segurança cirúrgica do paciente, desde $1990^{3}$ foi proposto que a Assistência de Enfermagem fosse realizada de forma sistematizada, através do processo de Enfermagem. As autoras propuseram um instrumento de coleta de informações do paciente cirúrgico denominado Sistematização de Assistência de Enfermagem Perioperatória (SAEP) ${ }^{3}$.

Segundo as mesmas, o instrumento deveria conter informações individuais do paciente, com dados de identificação, anamnese, exame físico e necessidades de cuidados de Enfermagem (diagnósticos de Enfermagem), além de intervenções e avaliação dos cuidados oferecidos ${ }^{4}$. O SAEP tem como objetivo garantir uma assistência planejada, com foco em todos os períodos operatórios (perioperatório), ou seja, desde o pré-operatório (quando o paciente é informado que precisará realizar um procedimento cirúrgico e é hospitalizado em um leito), o intraoperatório (cirurgia propriamente dita) e o pós-operatório, em que o paciente já realizou a cirurgia, mas pode apresentar complicações. Devido à sua importância, no ano de 2002, a Sistematização da Assistência de Enfermagem tornou-se uma exigência do Conselho Federal de Enfermagem ${ }^{5}$.

Deste modo, um instrumento de Enfermagem sistematizado com informações do período perioperatório pode auxiliar as Equipes de Enfermagem, Médica e todos os envolvidos no procedimento cirúrgico a garantir uma assistência segura para o paciente.

A realização bem sucedida de uma cirurgia requer conhecimento de anatomia e fisiologia, e das técnicas cirúrgicas e intervenções que previnam complicações perioperatórias. A Enfermagem em Unidade Cirúrgica, ao fornecer subsídios para a criação de um modelo assistencial, proporciona um avanço direcionado ao aprimoramento da assistência ao paciente perioperatório, à família e à comunidade ${ }^{4,5}$

Assim, o objetivo do presente estudo foi descrever a construção e a validação de conteúdo de um instrumento para registro da assistência de Enfermagem Perioperatória (SAEP) para o Centro Cirúrgico do Hospital das Clínicas de Rio Branco, no Estado do Acre, visando a atender às exigências da OMS propostas no segundo Desafio Global e reafirmadas no Manual Cirurgias Seguras Salvam Vidas do Ministério da Saúde.

\section{Método}

O presente estudo constitui-se de um relato de experiência.

O Hospital das Clínicas foi fundado em 1991, como hospital de especialidades, com seu estatuto social publicado no Diário Oficial do Estado do Acre (DOE Acre) no 5.594, de 13 de agosto de 1991. A Fundação Hospital Estadual do Acre, como era conhecida, foi constituída com personalidade jurídica de direito privado sem fins lucrativos. Tinha como finalidades prestar assistência médico-hospitalar e sanitária em todos os níveis de atenção à saúde, proporcionar treinamento a estudantes e técnicos, apoiar e promover a investigação epidemiológica, bem como cursos, seminários e pesquisa, e a divulgação de conhecimentos científicos, tornando-se um hospital-escola e de referência em saúde pública em Rio Branco e em todo o Estado.

O Centro Cirúrgico do Hospital das Clínicas do Acre tem oito salas cirúrgicas, realiza em média 40 cirurgias diariamente e já possui um instrumento para a coleta de dados assistenciais, porém, não sistematizado. 
A iniciativa deste trabalho surgiu da necessidade de auxiliar os profissionais do campo de estágio curricular do Curso de Graduação a desenvolver um instrumento de registro de ações de Enfermagem Perioperatória, embasado no conhecimento obtido no Congresso da Associação Brasileira de Enfermagem em Centro Cirúrgico (SOBECC), realizado em julho de 2011, na cidade de São Paulo-SP, do qual a orientadora deste projeto e os componentes da Equipe de Enfermagem do Centro Cirúrgico do Hospital das Clínicas participaram. Nesse momento, foi criada a parceria entre as duas instituições para a elaboração de um instrumento para registro da SAEP, conforme exigências do Conselho Federal de Enfermagem e do Manual Cirurgias Seguras Salvam Vidas.

Deste modo, a construção e a validação do instrumento foram realizadas através das seguintes fases:

- Aproximação entre a gerência de Enfermagem do Hospital das Clínicas, a Enfermeira responsável pelo Centro Cirúrgico e as pesquisadoras do Curso de Bacharelado em Enfermagem da UFAC, para averiguar a necessidade e o interesse da equipe em aderir ao objetivo do projeto.

- Revisão bibliográfica e construção do instrumento para registro da SAEP: a busca de artigos foi realizada através das palavras-chave: enfermagem perioperatória; diagnósticos de enfermagem; centro cirúrgico; cirurgias seguras; SAEP; assistência de enfermagem. Foram selecionados, para construção do instrumento, 19 artigos, por meio das bases de dados Scielo, Lilacs e BVS, além do Manual do Ministério da Saúde Brasileiro Cirurgias Seguras Salvam Vidas, que aborda a assistência de Enfermagem no período perioperatório e tem uma lista de verifícação de segurança cirúrgica. Livros específicos de centro cirúrgico e exame físico também foram utilizados, assim como o atual Instrumento de Enfermagem do Centro Cirúrgico.

Os referenciais teóricos selecionados tiveram como objetivos: i) levantar dados e informações relevantes sobre o exame físico do paciente cirúrgico para a construção da primeira fase do processo de Enfermagem (Histórico de Enfermagem) e ii) identificar os diagnósticos de Enfermagem mais frequentes nos períodos pré, intra e pós-operatório, para planejamento da assistência e prescrição do cuidado ao paciente cirúrgico. A teoria utilizada para nortear a SAEP foi a de Wanda de Aguiar Horta, com foco nas Necessidades Humanas Básicas (NHB).

- Avaliação e validação de conteúdo: após a construção do instrumento pelas pesquisadoras, o próximo passo foi a sua validação de conteúdo.

Foi realizado contato com a Enfermeira responsável pelo Centro Cirúrgico e com a gerência geral de Enfermagem do Hospital das Clínicas, para apresentar o protótipo do instrumento e agendar as aulas expositivas para capacitação da Equipe de Enfermagem sobre o tema, bem como a análise do instrumento.
As aulas foram realizadas nos dias 20/jul./2012, 02/ ago./2012 e 08/ago./2012, com duração total de 12 horas, nas quais foram abordados: o processo de Enfermagem; os principais diagnósticos de Enfermagem no período perioperatório; um caso cirúrgico prático para as Enfermeiras identificarem os diagnósticos de Enfermagem; o Segundo Desafio Global da OMS sobre segurança do paciente cirúrgico; o Manual Cirurgias Seguras Salvam Vidas e, por último, o instrumento desenvolvido, o qual foi distribuído para as Enfermeiras, as Técnicas de Enfermagem e os docentes avaliarem. Nesse momento, foi solicitado que os mesmos apontassem dúvidas e sugestões, bem como avaliassem o conteúdo do instrumento tendo como base o objetivo do estudo.

O instrumento foi validado por 12 Enfermeiras, 17 Técnicos de Enfermagem e dois docentes da área de Enfermagem em Centro Cirúrgico, todos com experiência profissional na área cirúrgica de, no mínimo, três anos, e no máximo de 20 anos.

Este projeto foi submetido e aprovado pelo Comitê de Ética da Universidade Federal do Acre sob o CAAE $\mathrm{n}^{\mathrm{o}}$ 02098112.3.0000.5010.

\section{Resultados}

O instrumento foi construído em duas folhas e meia de tamanho A4, constituído, em sua maioria, de perguntas em check list (Apêndice 1), o que permite ao profissional um direcionamento na prestação de serviços ao paciente cirúrgico. Os itens foram distribuídos em sete partes: A) Identificação do paciente; B) Consulta pré-operatória (setor de internação); C) Período pré-operatório imediato (enfermaria); D) Período pré-operatório imediato (Centro Cirúrgico); E) Período intraoperatório (SO); F) Período pós-operatório imediato (Sala de recuperação pós-anestésica - SRPA), e G) Pós-operatório imediato (enfermaria).

Na primeira parte, 'identificação do paciente', constam informações de identificação, como nome, data da internação e dados sociodemográficos. Aúnica sugestão das avaliadoras foi mudar a palavra 'matrícula do paciente' por 'registro'.

Em relação à 'consulta pré-operatória', acreditava-se que a mesma ocorria na Enfermaria Cirúrgica, porém, foi informado pelas avaliadoras que esta acontecia, primeiramente, no Setor de Internação. Essa modificação foi realizada, assim como a retirada dos itens 'punção venosa' e 'tricotomia', visto que os mesmos são realizados na enfermaria.

Durante a avaliação, questionou-se se os diagnósticos de Enfermagem presentes no pré-operatório teriam de ser julgados pelo Enfermeiro do Setor de Internação ou pelo Enfermeiro Cirúrgico da enfermaria. O consenso foi que estes devem ser realizados no Setor de Internação devido à avaliação abrangente (histórico de enfermagem realizada neste setor, que inclui a entrevista e o exame físico céfalocaudal), que contém informações para o julgamento dos problemas de enfermagem e elaboração dos diagnósticos de 
enfermagem. No entanto, a continuidade das intervenções prescritas em relação aos diagnósticos listados deve ser realizada pelos dois setores.

Quanto ao conteúdo do histórico, que inclui a entrevista e o exame físico, este não foi alterado, sendo avaliado como prático, informativo e de fácil utilização pelos profissionais que participaram do processo de validação.

Foi incluído o item 'Pré-operatório imediato - enfermaria', porque o paciente, após receber a primeira avaliação no setor de internação, é encaminhado para a enfermaria cirúrgica. Conforme sugestão das Enfermeiras, foram incluídos: cirurgia proposta; sinais vitais; local da punção venosa; número do jelco utilizado na punção venosa; tricotomia, e a data e a hora em que o paciente foi encaminhado ao Centro Cirúrgico.

O período pré-operatório imediato, realizado no Centro Cirúrgico, consta de informações relevantes, como data e hora de admissão no Centro Cirúrgico; confirmação da identidade do paciente, assim como do sítio cirúrgico, e procedimentos a serem realizados (listagem do manual de cirurgias seguras), exames pré-operatórios, comorbidades, alergias, jejum, uso de prótese dentária, sinais vitais, presença de sondas, punção venosa, número da sala operatória e horário em que o paciente será encaminhado. Foi solicitada a inclusão da avaliação do nível de consciência e da integridade da pele do paciente, visto que são admitidos pacientes debilitados. Após correções, o instrumento foi considerado prático e de fácil execução, sendo útil para lembrar todas as questões necessárias para a segurança do paciente.

Na quinta parte, 'entrada no Centro Cirúrgico', intraoperatório, o instrumento visa a obter informações da listagem de verificação de segurança cirúrgica do Manual de Cirurgias Seguras do Ministério da Saúde, que devem ser checadas antes da indução anestésica (sign in), antes da incisão cirúrgica (time out) e antes de o paciente sair da sala operatória (sign out). Os itens sugeridos e incluídos nesta parte foram: cirurgia proposta, nome do Cirurgião, do Auxiliar de Cirurgia, do Instrumentador e do circulante.

Os diagnósticos de Enfermagem (DE) presentes no transoperatório foram aceitos; porém, foi sugerida a colocação de espaços extras para a inclusão, se necessário, de algum DE não ter sido elencado no check list. O julgamento dos DE são de responsabilidade das Enfermeiras. Atualmente, o Centro Cirúrgico conta com três Enfermeiras assistenciais por plantão, além da Enfermeira coordenadora.

As avaliadoras disseram que os diagnósticos propostos e as intervenções, na maioria das vezes, já são realizados pela equipe; porém, sem as anotações que comprovem a sua execução, reforçando que o instrumento ajudará a formalizar e registrar a assistência de Enfermagem.

Já na sexta parte do instrumento, o 'pós-operatório imediato', a recuperação pós-anestésica, constam informações da sala de recuperação pós-anestésica (SRPA). Em relação ao instrumento vigente usado no Centro Cirúrgico, foi introduzida a Escala de Aldrete-Kroulik, essencial para a alta do paciente, com os sinais vitais com espaço para avaliação de 15 em 15 minutos na primeira hora e, a cada 30 minutos, na segunda hora, conforme preconizado. Os diagnósticos de Enfermagem foram bem aceitos.

As sugestões deste período foram incluir volume de líquidos infundidos, volume dos drenos e da diurese, e hora da saída da SRPA, as quais foram prontamente atendidas. A Enfermeira responsável pela SRPA achou o instrumento passível de realização e estruturado.

Na sétima e última parte, o 'pós-operatório imediato', realizado na enfermaria, segundo sugestão, foi introduzido o horário de entrada na enfermaria, além do registro dos sinais vitais, do nível de consciência, das queixas, da hidratação venosa e das condições do curativo.

\section{Discussão}

$\mathrm{Na}$ medida em que a sistematização de assistência de Enfermagem Perioperatória (SAEP) é um modelo assistencial que favorece a garantia da qualidade ao paciente cirúrgico por ser uma proposta organizada de planejamento, com o objetivo de promover a assistência integral e continuada ao paciente cirúrgico, observou-se a necessidade de aperfeiçoamento do instrumento de coleta de dados já existente, devido à escassez de informações inerentes ao paciente cirúrgico, como dados sociodemográficos e de histórico (entrevista e exame físico). Observe-se que o instrumento de coleta não abrangia as fases necessárias do processo de Enfermagem proposto por Wanda Horta ${ }^{4,6,7}$.

A Resolução do Conselho Federal de Enfermagem, COFEN - 272/2002 ${ }^{8}$, dispõe sobre a obrigatoriedade da SAE no Brasil, como atividade privativa do Enfermeiro, mas deve participar desse processo toda a Equipe de Enfermagem, uma vez que todos trabalham em prol do paciente. Notouse que mesmo decorridos dez anos dessa resolução, as recomendações não haviam sido incorporadas no hospital do estudo.

É importante salientar que, na elaboração de um instrumento, certos itens, como a identificação completa do paciente, com seu número de registro hospitalar, local, a identificação do profissional que prestou assistência ao paciente, assim como o horário e a data em que esta ocorreu são primordiais. Nesse contexto, destaca-se a importância dos registros de forma cronológica para explicar os eventos que ocorreram com o paciente durante o período perioperatório, além de alimentar bancos de dados e solicitações para a melhoria da qualidade do serviço e proporcionar o respaldo legal dos profíssionais e da instituição de saúde ${ }^{4}$.

Para a construção do instrumento para registro da SAEP do Hospital das Clínicas, optou-se por utilizar somente duas folhas e meia, de modo que este não ficasse muito extenso e de difícil utilização, otimizando, assim, tempo e espaço para o seu preenchimento. Uma das dificuldades encontradas na construção foi condensar todo o conteúdo dentro de um 
pequeno espaço sem deixar lacunas com relação ao conteúdo necessário para o adequado registro das ações.

Dividido em sete partes, o instrumento foi considerado de fácil utilização, uma vez que cada profissional é responsável pelo preenchimento das informações pertinentes ao seu respectivo setor de atendimento ao paciente, podendo preenchê-lo de forma rápida, já que o mesmo é disposto de forma simples e prática (check list).

Na recepção do paciente, que ocorre no setor de internação, o exame físico céfalo-caudal deve ser realizado pelo Enfermeiro, averiguando as condições dos sistemas orgânicos e a presença de sondas, drenos, infusões intravenosas e limitações, sendo que a expressão de algum desconforto deve ser valorizada. A entrevista realizada pelo Enfermeiro no momento pré-operatório é de suma importância, pois é nesse momento que se identificam as necessidades individuais para cada paciente, atentando para pontos relacionados, como a existência de alergias, tabagismo, alcoolismo e comorbidades, além dos diagnósticos específicos do pré-operatório ${ }^{5,69}$.

No período intraoperatório, em que se rompem as barreiras epiteliais, é o período em que o paciente fica mais exposto aos patógenos que podem levar a complicações e até mesmo à morte. Por esse motivo, enfatizou-se a lista de verificação de segurança cirúrgica da OMS, que traz itens essenciais para a observação do paciente cirúrgico e os diagnósticos específicos deste período ${ }^{1,10-16}$

O pós-operatório é uma fase crítica para o paciente, em que ele fica vulnerável a diversas complicações, principalmente as de origem respiratória, circulatória e gastrointestinal. Por essa razão, ao admitir o paciente na recuperação pós-anestésica, é necessário que a avaliação inicial do Enfermeiro consista em inspecionar prontamente o paciente, monitorizando-o de forma que se avaliem todos os parâmetros vitais, além da realização do exame físico céfalo-caudal, com ênfase no local cirúrgico.

Neste período, é necessário também atentar para a reposição de líquidos, o débito urinário e gástrico, a avaliação de curativos, os cateteres e drenos, com o objetivo de prevenir ou tratar complicações, tendo sido elaborados os diagnósticos específicos para este período, conforme revisão da literatura ${ }^{16-20}$.

O índice de Aldrete-Kroulik - que era inexistente no instrumento anterior do referido hospital - é utilizado em pacientes submetidos ao ato anestésico cirúrgico, como respaldo científico na avaliação da alta do paciente da SRPA $^{19}$.

As avaliadoras se empenharam nas aulas, auxiliaram nas modificações do SAEP e ficaram agradecidas ao ver, finalmente, a sistematização saindo da teoria para atender à prática. Muitas verbalizaram que o processo de Enfermagem agora não é mais tão difícil como imaginavam e destacaram a importância de utilizarem um instrumento que valorize a prática de Enfermagem, assim como garanta maior atenção à segurança do paciente cirúrgico.
A dificuldade encontrada pelas pesquisadoras foi em relação à realização das aulas de treinamento, visto que era difícil a liberação de muitos funcionários por turno; por esta razão, as aulas foram realizadas em três momentos e em turnos diferentes, e até mesmo dentro do Centro Cirúrgico.

Os dados obtidos em todo o período perioperatório devem ser registrados de forma clara e concisa, e em impresso próprio, com informações pertinentes ao paciente cirúrgico de modo que estas direcionem os cuidados da Equipe de Enfermagem ${ }^{19}$. Deste modo, o SAEP contribuirá para a sistematização da assistência cirúrgica e a segurança do paciente.

\section{Conclusão}

Esta pesquisa foi iniciada com o objetivo de melhorar a assistência e a segurança do paciente cirúrgico no Hospital das Clínicas do Acre, devendo, para tanto, realizar a construção e a validação do instrumento de Sistematização da Assistência de Enfermagem Perioperatória (SAEP). Todos os passos desta pesquisa só foram possíveis devido à aceitação e à confiança da gerência do hospital, da Equipe de Enfermagem do Centro Cirúrgico e das Enfermarias, em relação à proposta das pesquisadoras.

$\mathrm{O}$ instrumento de registro da SAEP foi analisado como satisfatório pelas avaliadoras por ser de fácil interpretação e preenchimento. Todas as sugestões de introdução e correção de itens foram discutidas e, quando aceitas, incluídas no instrumento.

Com o trabalho finalizado, percebemos o quão importante foi a parceria da academia com o serviço de saúde, nosso campo de prática. Sugerimos que mais trabalhos como este sejam realizados no intuito de auxiliar os campos de estágio e envolver os alunos, e que tais trabalhos resultem em pesquisas que atenderão as reais necessidades do serviço e possibilitem a valorização da nossa profissão e a segurança do paciente.

O próximo passo desta pesquisa é a realização da análise das propriedades psicométricas do instrumento para a SAEP por testes estatísticos, assim como o acompanhamento da implementação do mesmo.

\section{Referências}

1. Organização Mundial da Saúde - OMS. Segundo desafio global para a segurança do paciente: Manual - cirurgias seguras salvam vidas. Rio de Janeiro: Organização Pan-Americana da Saúde, Ministério da Saúde, Agência Nacional de Vigilância Sanitária; 2009.

2. Ferraz EM. A cirurgia segura: uma exigência do século XXI. Rev Col Bras Cir. 2009;36(4):281-2. PMid:20076914. http://dx.doi. org/10.1590/S0100-69912009000400001

3. Castellanos BEP, Jouclas VMG. Assistência de enfermagem perioperatória: num modelo conceitual. Rev Esc Enferm USP. 1990;24(3):359-70. PMid:2082439.

4. Fonseca RM, Peniche ACG. Enfermagem em centro cirúrgico: trinta anos após criação do Sistema de Assistência de Enfermagem 
Perioperatória. Acta Paul Enferm. 2009;22(4):428-33. http:// dx.doi.org/10.1590/S0103-21002009000400013

5. Sociedade Brasileira de Enfermeiros de Centro Cirúrgico, Recuperação Anestésica e Centro de Material e Esterilização - SOBECC. Práticas recomendadas SOBECC: centro cirúrgico, recuperação anestésica e centro de material e esterilização. 5. ed. São Paulo: SOBECC; 2009.

6. Santos RR, Piccoli M, Carvalho ARS. Diagnósticos de enfermagem emocionais identificados na visita pré-operatória em pacientes de cirurgia oncológica. Cogitare Enferm. 2007;12(1):52-61.

7. Horta WA. O processo de enfermagem. São Paulo: EPU, EDUSP; 1979.

8. Conselho Federal de Enfermagem - COFEN. Resolução 272/2002 de 27 de agosto de 2002. Rio de Janeiro: COFEN; 2002 [acesso em 2012 Ago 11]. Disponível em: www.portalcofen.gov.br/node/4309.

9. Lopes AER, Pompeo DA, Canini SRMS, Rossi LA. Nursing diagnoses of patients in the preoperative period of esophageal surgery. Rev Latino-Am Enfermagem. 2009;17(1):66-73. http:// dx.doi.org/10.1590/S0104-11692009000100011

10. Suriano ML, Barros ALBL. Identificação dos diagnósticos de enfermagem mais freqüentes no período perioperatório dos pacientes submetidos a cirurgias cardiovasculares. Acta Paul Enf. 2000;13(2):98-104.

11. Piccoli M, Matos FGOA. Sistematização da assistência de enfermagem perioperatória. In: Seminário Nacional Estado e Políticas Sociais no Brasil; 2003; Cascavel. Cascavel: Edunioeste; 2003.

12. Bedin ER, Miranda LB, Barreto RASS. Humanização da assistência de enfermagem em centro cirúrgico. Rev. Eletrônica
Enferm. 2005;7(1):118-27 [acesso em 2011 Out 23]. Disponível em www.fen.ufg.br/revista.html.

13. Galdeano LE, Rossi LA, Nobre LF, Ignácio DS. Nursing diagnosis in the intra-operative period of cardiac surgery. Rev Latino-Am Enfermagem. 2003;11(2):199-206. http://dx.doi.org/10.1590/ S0104-11692003000200009

14. Flório MCS, Galvão CM. Surgery in outpatient units: identification of nursing diagnoses in the perioperative period. Rev LatinoAm Enfermagem. 2003;11(5):630-7. http://dx.doi.org/10.1590/ S0104-11692003000500010

15. Soares LH, Pinelli FGS, Abrão ACFV. Construção de um instrumento de coleta de dados de enfermagem em ginecologia. Acta Paul Enferm. 2005;18(2):156-64. http://dx.doi.org/10.1590/ S0103-21002005000200007

16. Galdeano LE, Rossi LA. Elaboration and validation of data collection instruments for the perioperative period of cardiac surgery. Rev Latino-Am Enfermagem. 2002;10(6):800-4. PMid:12696551.

17. Araújo EAG, De Paula KA, Oliveira LAF, Arruda ACV. Sistematização da assistência de enfermagem na sala de recuperação pós-anestésica. Rev SOBECC. 2011;16(3):43-51.

18. Carvalho R, Bianchi ER. Enfermagem em centro cirúrgico e recuperação. Barueri: Manole; 2007.

19. Possari JF. Assistência de Enfermagem na Recuperação pósanestésica (RPA). São Paulo: Iátria; 2003.

20. Ribeiro MB, Bonfim IM, Silveira CT. Estratégias de capacitação da equipe de enfermagem de centro cirúrgico oncológico. Rev SOBECC. 2011;16(3):21-9. 


\section{APÊNDICE I - SAEP \\ HOSPITAL DAS CLÍNICAS DE RIO BRANCO \\ SAEP - SISTEMATIZAÇÃO DA ASSISTÊNCIA DE ENFERMAGEM PERIOPERATÓRIA}

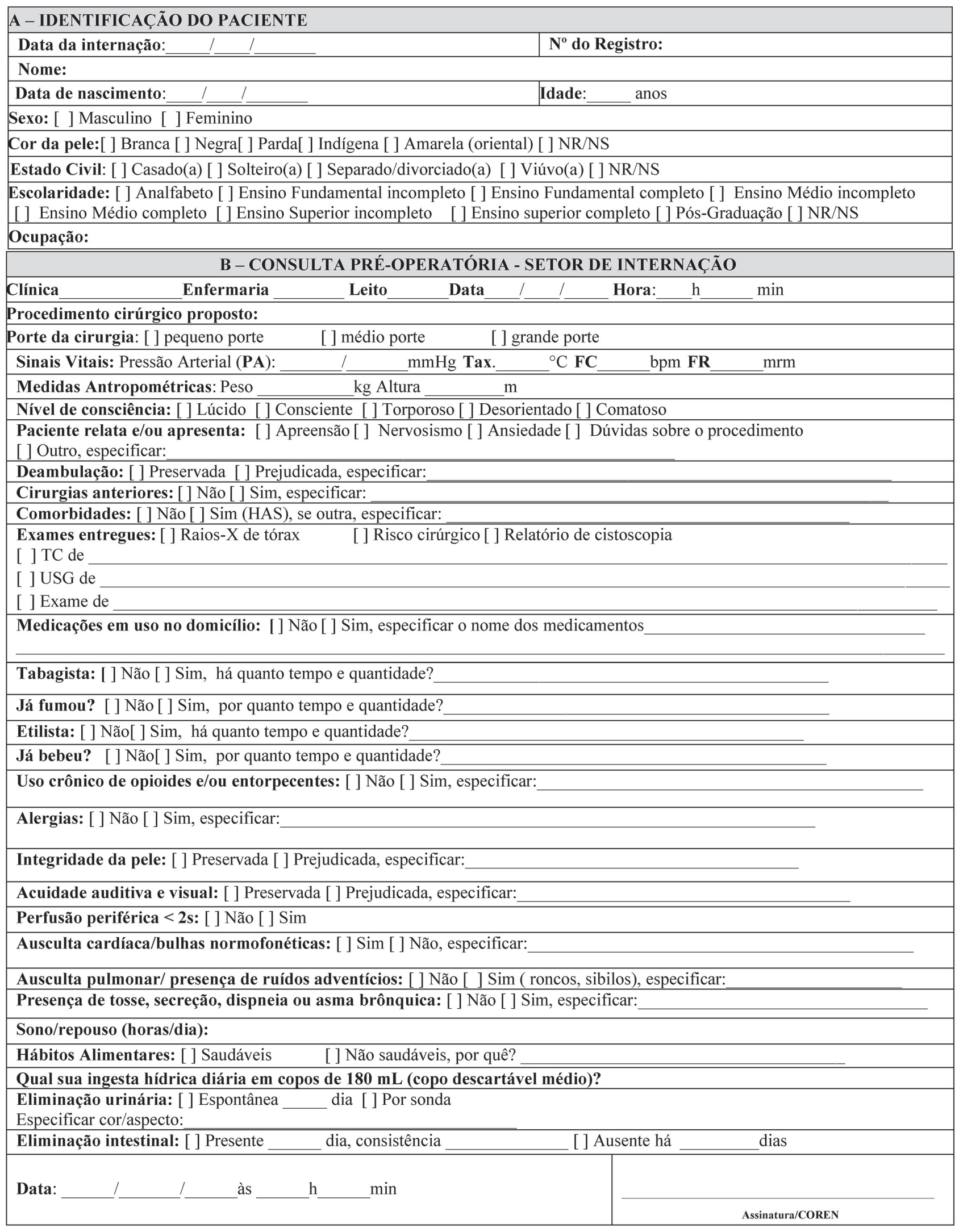




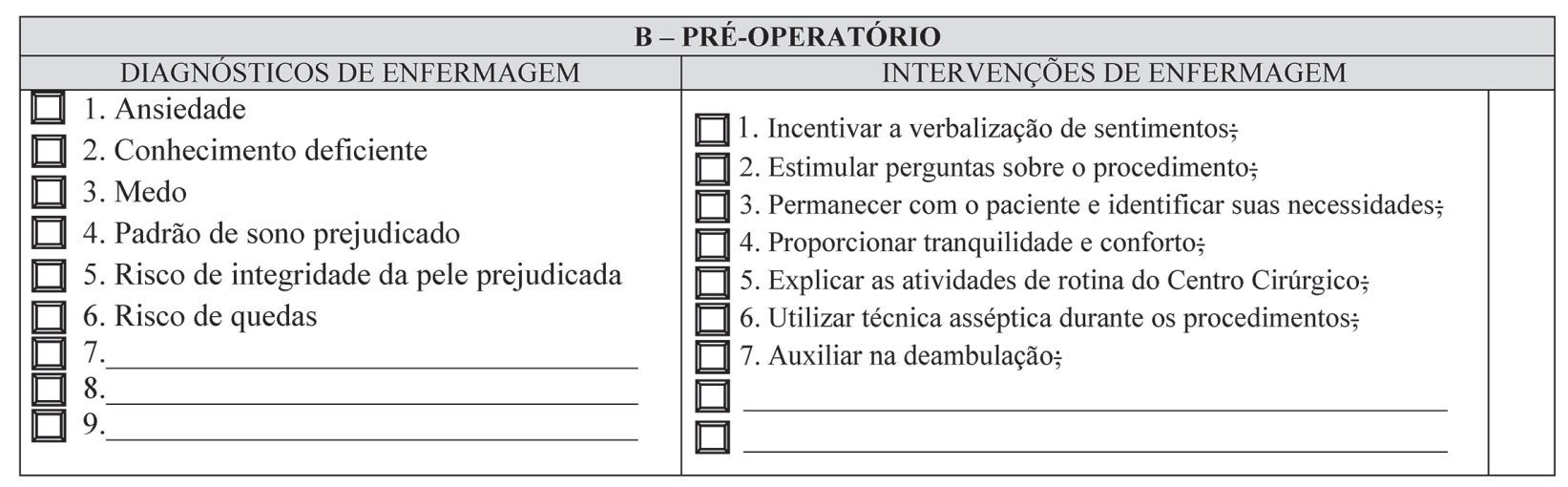

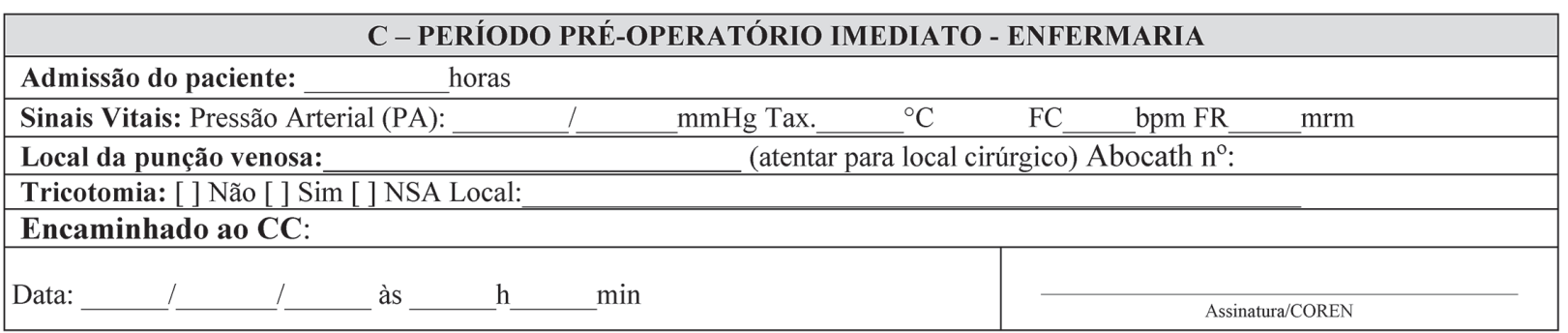

\begin{tabular}{|c|c|}
\hline \multicolumn{2}{|c|}{ D - PERÍODO PRÉ-OPERATÓRIO IMEDIATO - ENTRADA NO CENTRO CIRÚRGICO } \\
\hline Hora de entrada no C.C: & \\
\hline \multicolumn{2}{|l|}{ Paciente: [ ] Deambulando [ ] Transportado em cadeira de rodas/maca } \\
\hline \multicolumn{2}{|c|}{ Exames pré-operatórios (de imagem, de risco cirúrgico, laboratoriais): [ ] Não [ ] Sim } \\
\hline \multicolumn{2}{|c|}{ Paciente confirmou identidade, sítio cirúrgico, procedimento e consentimento [ ] Não[ ] Sim } \\
\hline \multicolumn{2}{|l|}{ Comorbidades: [ ] Não [ ] Sim (HAS). Se outra, especificar: } \\
\hline \multicolumn{2}{|l|}{ Alergias: [ ] Não [ ] Sim, especificar: } \\
\hline \multicolumn{2}{|l|}{ Jejum: [ ] Não [ ] Sim } \\
\hline \multicolumn{2}{|c|}{ Prótese dentária, lentes e/ou adornos: [ ] Não [ ] sim, foi retirado, e identificado e encaminhado para: } \\
\hline \multicolumn{2}{|c|}{ Sinais Vitais: Pressão Arterial (PA): ___ mmHg Tax.___ } \\
\hline \multicolumn{2}{|l|}{ Portando sondas: [ ] Não [ ] Sim, especificar: } \\
\hline \multicolumn{2}{|l|}{ Punção venosa viável: [ ] Sim [ ] Não, foi realizada uma nova. Local } \\
\hline Encaminhado a S.O $\mathbf{n}^{\circ} . \quad$ Hora: $\_$_ & Assinatura/COREN \\
\hline
\end{tabular}

\begin{tabular}{|c|}
\hline E - PERÍODO INTRAOPERATÓRIO - CENTRO CIRÚRGICO \\
\hline ANTES DA INDUÇÃO ANESTÉSICA \\
\hline Cirurgia Proposta: \\
\hline Paciente confirmou identidade, sítio cirúrgico, procedimento e consentimento? [ ] Não [ ] Sim \\
\hline Sítio demarcado: [ ] Não[ ] Sim [ ] Não se aplica \\
\hline Verificação de segurança anestésica concluída: [ ] Não [ ] Sim \\
\hline Oxímetro de pulso no paciente e em funcionamento? [ ] Não [] Sim \\
\hline Via aérea difícil/risco de aspiração? [ ] Não [] Sim, e equipamento/assistência disponíveis \\
\hline $\begin{array}{l}\text { Risco de perda sanguínea }>500 \mathrm{~mL}(7 \mathrm{~mL} / \mathrm{kg} \text { em crianças)? [ ] Não[ ] Sim, e acesso endovenoso adequado e planejamento para } \\
\text { fluidos. }\end{array}$ \\
\hline Início da anestesia: $\quad \mathrm{h} \quad \min ^{*}$ Tipo de anestesia realizada: \\
\hline Anestesista: \\
\hline ANTES DA INCISÃO CIRÚRGICA \\
\hline $\begin{array}{l}\text { Cirurgião, Anestesiologista e a Equipe de Enfermagem confirmam verbalmente: identificação do paciente, sítio cirúrgico e } \\
\text { procedimento [ ] Sim [ ] Não, especificar }\end{array}$ \\
\hline A profilaxia antimicrobiana foi realizada nos últimos 60 min? [ ] Não [ ] Sim [ ] Não se aplica \\
\hline As imagens essenciais estão disponíveis? [ ] Não [ ] Sim ～[ ] Não se aplica \\
\hline $\begin{array}{l}\text { Posição do paciente para a cirurgia: [ ] Decúbito dorsal [ ] Decúbito Lateral [ ]Decúbito Ventral [ ] Semifowler [ ] Fowler } \\
\text { [ ] Trendelenburg [ ] Trendel.Invertido [ ] Litotômica [ ] Posição de canivete [ ] Posição genupeitoral [ ] Posição renal }\end{array}$ \\
\hline Utilização do Bisturi elétrico/laser: [ ] Não [ ] Sim, especificar \\
\hline Local da placa: \\
\hline Solução utilizada para degermação e antissepsia do campo operatório: \\
\hline
\end{tabular}




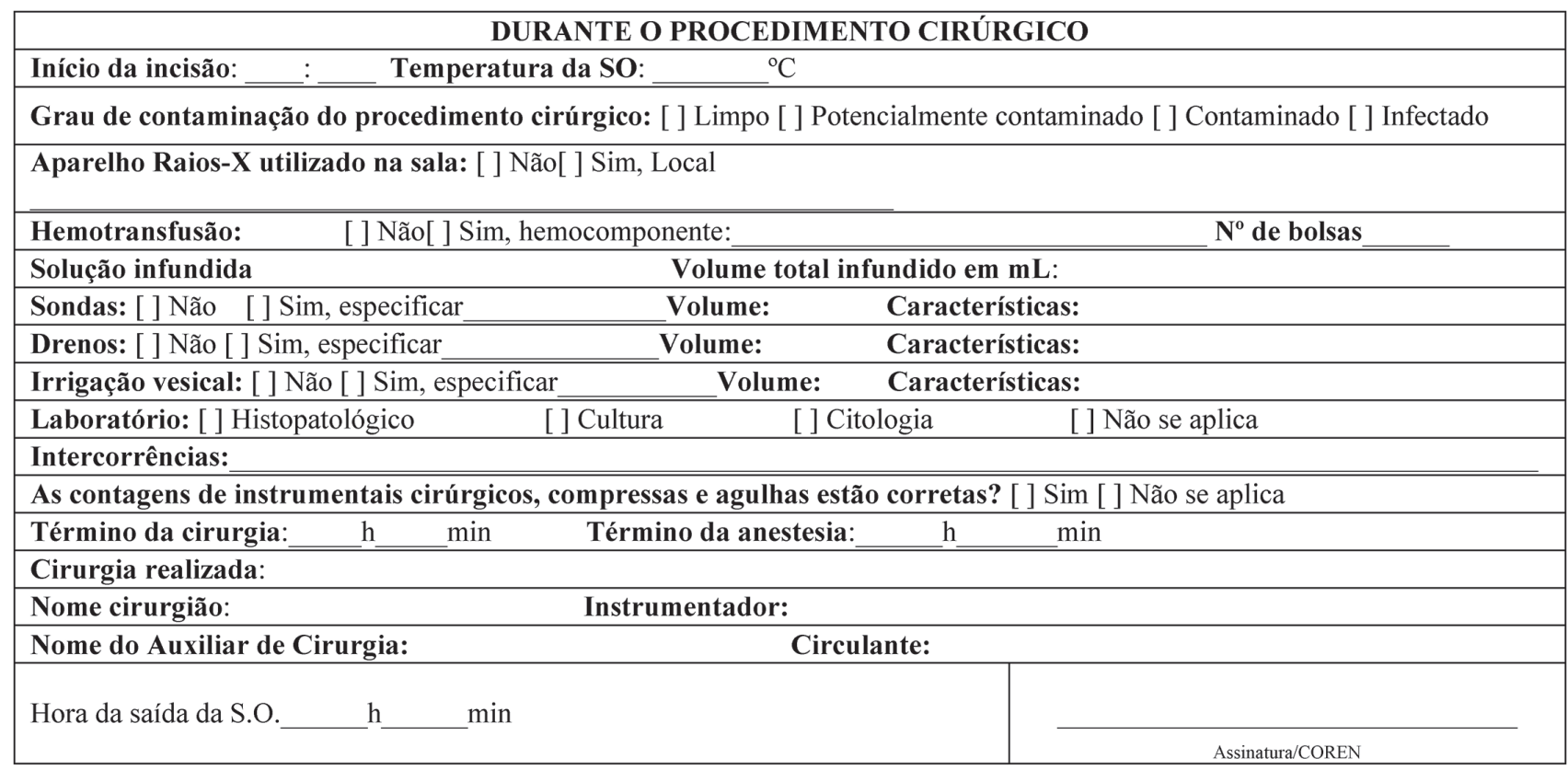

\begin{tabular}{|c|c|}
\hline \multicolumn{2}{|c|}{ E - INTRAOPERATÓRIO } \\
\hline DIAGNÓSTICOS DE ENFERMAGEM & INTERVENÇÕES DE ENFERMAGEM \\
\hline 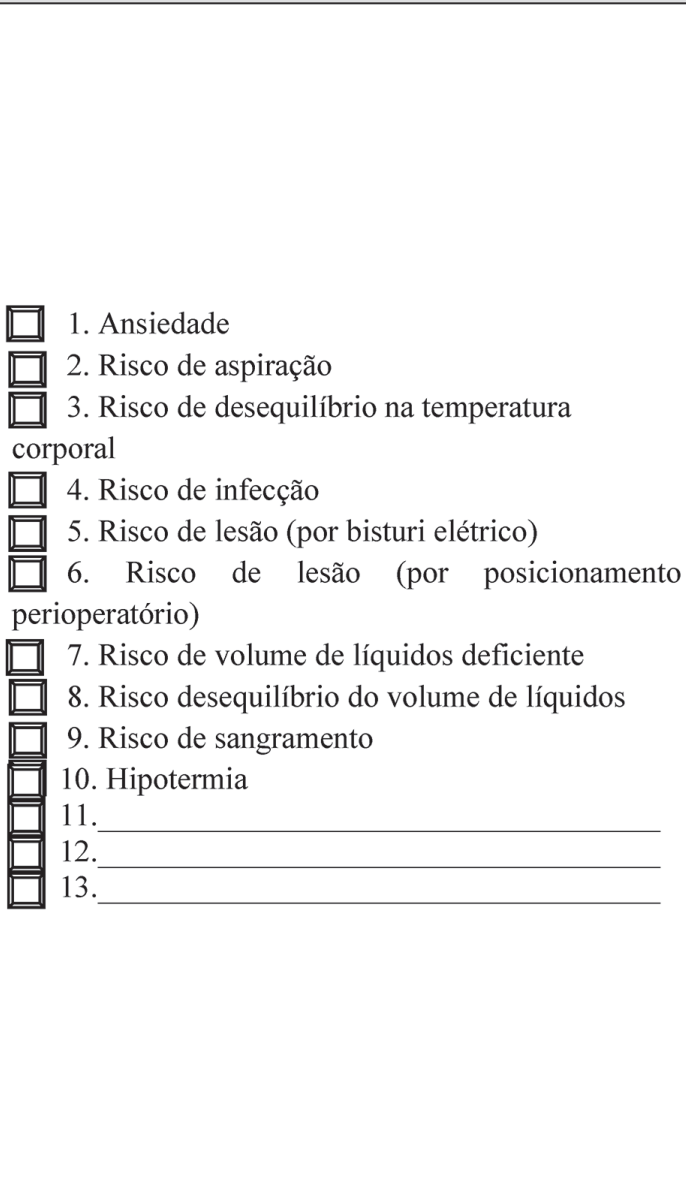 & 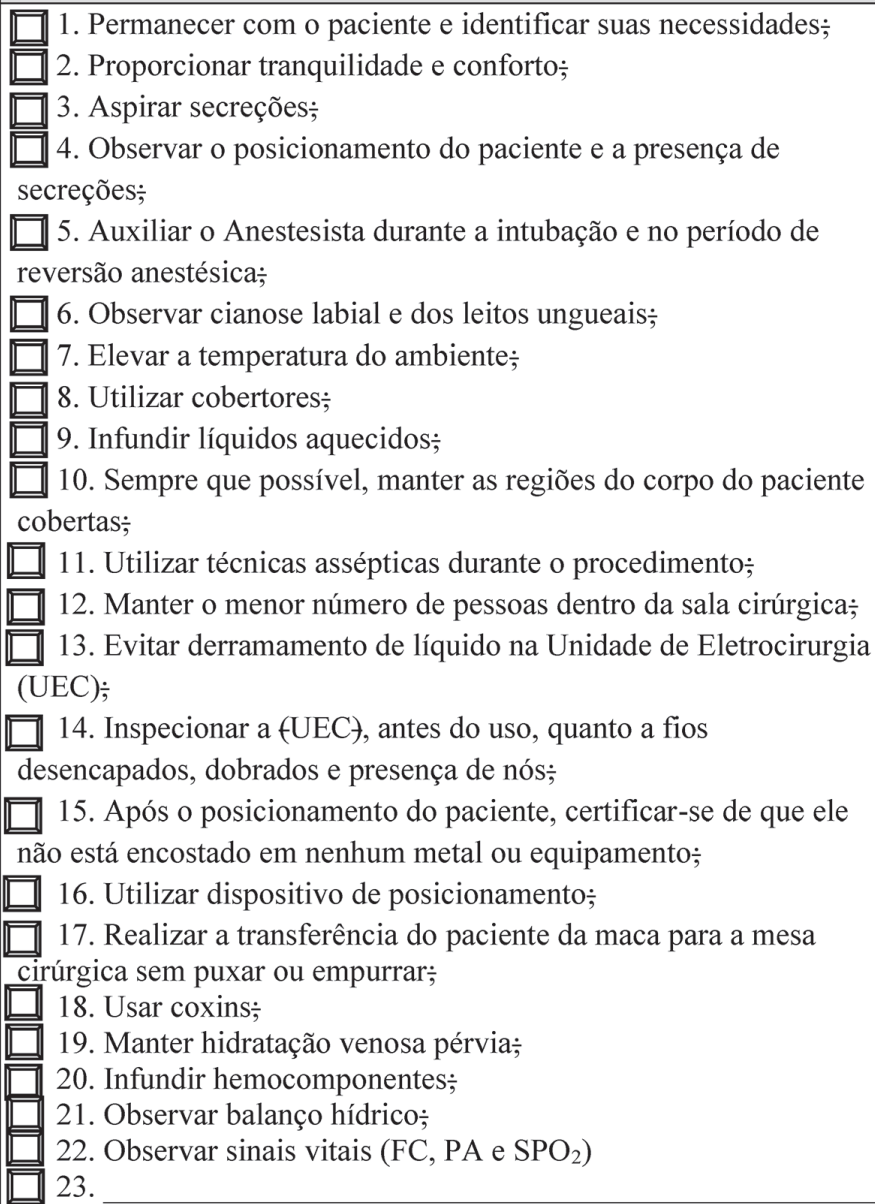 \\
\hline
\end{tabular}




\section{F - SALA DE RECUPERAÇÃO PÓS-ANESTÉSICA - SRPA}

Admissão do paciente: $\mathrm{h} \quad \min$ Acompanhado por:

Nível de consciência: [ ] Lúcido [ ] Sonolento [ ] Consciente [ ] Torporoso [ ] Desorientado [ ] Comatoso [ ] Agitado

[] Outro, especificar

Queixas: [ ] Dor [ ] Êmese [ ] Naúseas [ ] Frio [ ] Dispneia [] Tonturas [ ] Outras, especificar

[] Sem queixas

Hidratação venosa/local:

Solução infundida:

Condições:

Curativo cirúrgico/local:

Volume total infundido:

$\mathrm{mL}$

Sinais de hemorragia: [ ] Não [ ] Sim, especificar

Extremidades: [ ] Aquecidas [] Frias [ ] Cianóticas [] Perfundidas

Drenos: [ ] Não [ ] Sim, especificar

Diurese: [ ] Espontânea [] Ausente [ ] Por sonda vesical

Intercorrências:

\begin{tabular}{|c|c|c|c|c|c|c|c|}
\hline \multirow{5}{*}{ 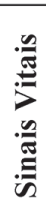 } & Parâmetro/ Hora & $15^{\prime}$ & 30 ' & $45^{\prime}$ & $60^{\prime}$ & $1 \mathrm{~h} 30$ & $2 \mathrm{~h}$ \\
\hline & Pressão Arterial & & & & & & \\
\hline & Pulso/FC & & & & & & \\
\hline & Temperatura & & & & & & \\
\hline & $\mathrm{SPO}_{2}$ & & & & & & \\
\hline
\end{tabular}

ESCALA DE ALDRETE E KROULIK

\begin{tabular}{|c|c|c|c|c|c|c|c|}
\hline Parâmetro & Resposta & Pontos & 15 & 30 & 45 & 60 & 1h30' \\
\hline \multirow{3}{*}{$\begin{array}{l}\text { Atividade } \\
\text { muscular }\end{array}$} & Movimenta os quatros membros & 2 & & & & & \\
\hline & Movimenta dois membros & 1 & & & & & \\
\hline & Incapaz de mover os membros voluntariamente ou sob comando & 0 & & & & & \\
\hline \multirow{3}{*}{ Respiração } & Capaz de respirar pronfundamente & 2 & & & & & \\
\hline & Dispneia ou limitação da respiração & 1 & & & & & \\
\hline & Apneia & 0 & & & & & \\
\hline \multirow{3}{*}{ Circulação } & PA com variação de até $20 \%$ do nível pré-anestésico & 2 & & & & & \\
\hline & PA com variação de 20 a 49\% do nível pré-anestésico & 1 & & & & & \\
\hline & PA com variação acima de $50 \%$ do nível pré-anestésico & 0 & & & & & \\
\hline \multirow{3}{*}{ Consciência } & Lúcido e orientado no tempo e no espaço & 2 & & & & & \\
\hline & Desperta, se solicitado & 1 & & & & & \\
\hline & Não responde & 0 & & & & & \\
\hline \multirow{3}{*}{ Saturação de $\mathrm{O2}$} & $\begin{array}{l}\text { Capaz de manter saturação de } \mathrm{O} 2>92 \% \text {, respirando em ar } \\
\text { ambiente }\end{array}$ & 2 & & & & & \\
\hline & Necessita de $\mathrm{O} 2$ para manter a saturação $>90 \%$ & 1 & & & & & \\
\hline & Saturação de $\mathrm{O} 2<90 \%$, com suplementação de oxigênio & 0 & & & & & \\
\hline Total de pontos & Obs.: de 8 a 10 pontos, paciente apto para alta. & - & & & & & \\
\hline
\end{tabular}




\begin{tabular}{|c|c|}
\hline \multicolumn{2}{|c|}{ F - SALA DE RECUPERAÇÂO PÓS-ANESTÉSICA } \\
\hline DIAGNÓSTICOS DE ENFERMAGEM & INTERVENÇÕES DE ENFERMAGEM \\
\hline 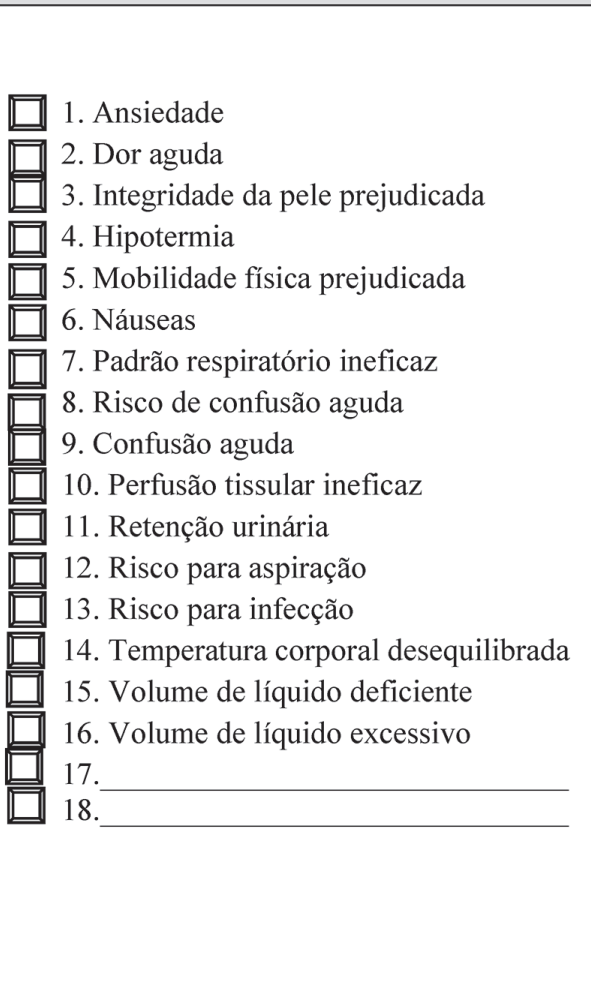 & 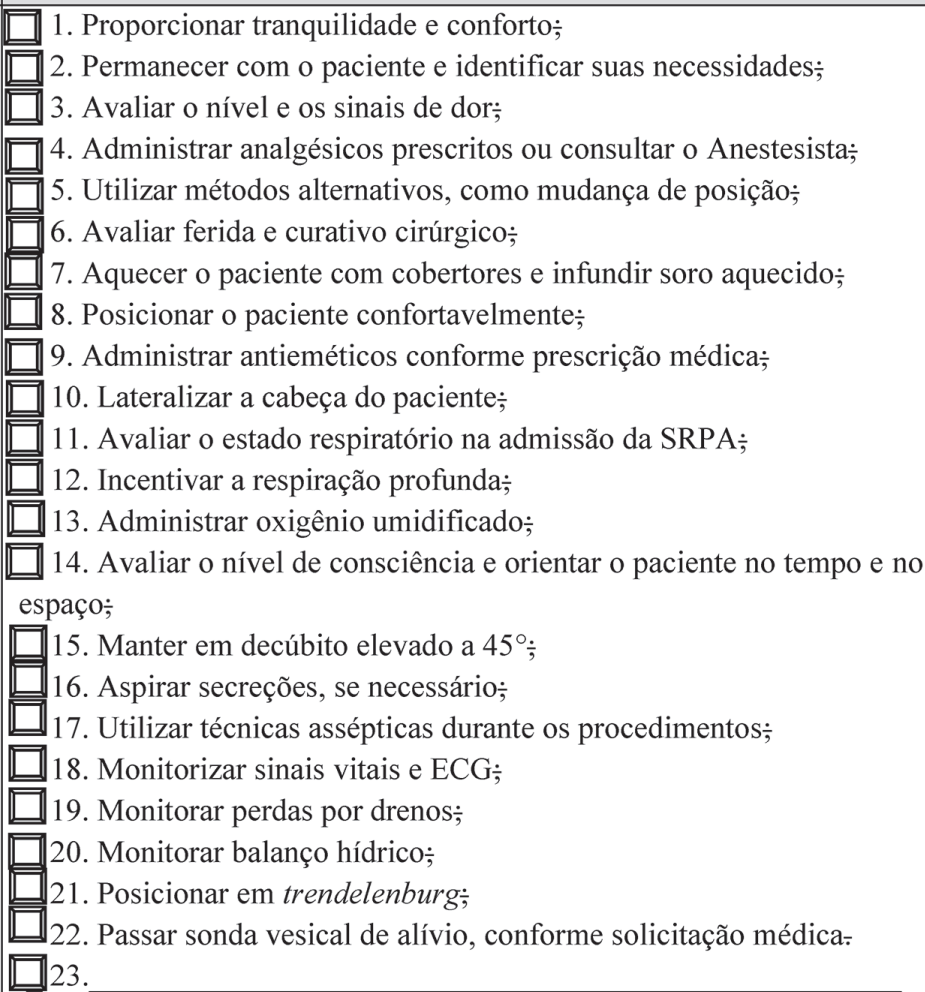 \\
\hline $\begin{array}{l}\text { Hora da saída da SRPA_ } \\
\text { Liberado por: } \\
\text { [ ] Entregue receita, atestado, retorno, espe }\end{array}$ & Assinatura/COREN \\
\hline
\end{tabular}

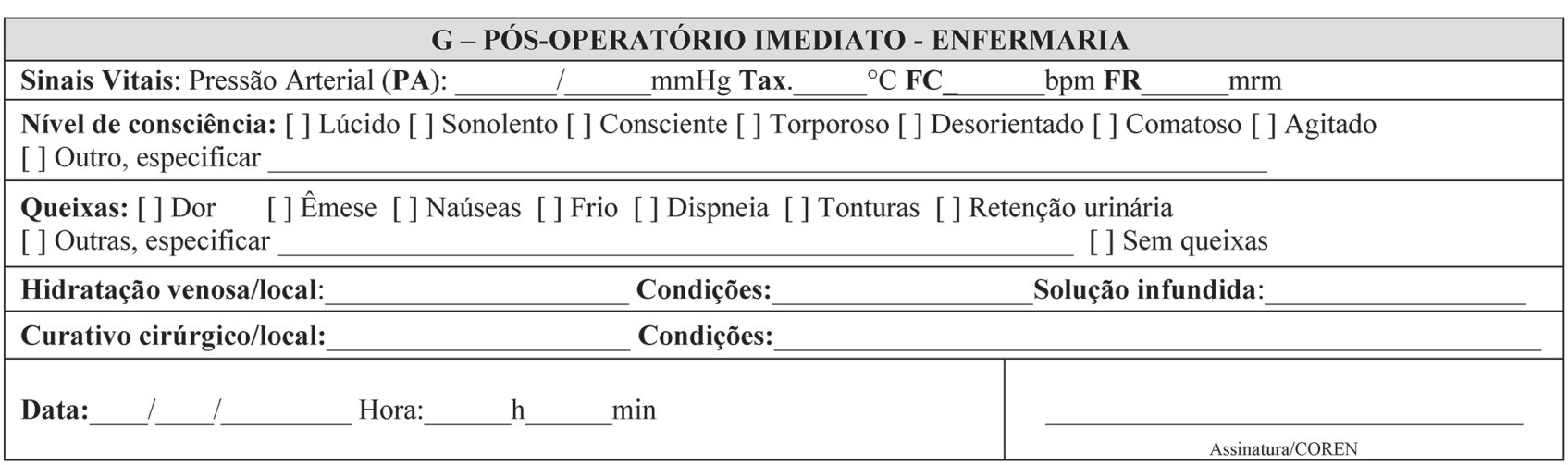

